\subsection{1}

doi: 10.15789/2220-7619-2018-4-9.31

\section{FERTILIZATION FAILURE IN HEIFERS INFECTED \\ BY UREAPLASMA DIVERSUM}

A.N. Vaganova, S.V. Borisenko, O.A. Freylikhman,

V.V. Roca, V.N. Verbov

St. Petersburg Pasteur Institute, St. Petersburg, Russia

Ureaplasma diversum is an opportunistic pathogen in cattle, but colonization of the respiratory tract by this ureaplasma, and its carriage in the reproductive tract may lead to the serious diseases. It also may be the cause of abortion and stillbirth in cattle.

The aim of this study was to estimate the fertilization effectiveness of in heifers into relation with $U$. diversum carriage in the vulval vestibule. All 20 heifers in the study group were from the same dairy farm from Leningradskaya oblast. The samples were collected from the vulval vestibule by cotton swab. At the sample collection vulvar mucous appearance was estimated. The $U$. diversum carriage was detected by real-time PCR assay with diagnostic system "Ureaplasma diversum Amp" (St. Petersburg Institut Pasteur, Russia).

In the group of 20 heifers, 13 had symptoms or granular vulvovaginitis, including yellowish-gray pustules on the mucous. No other reproductive disease symptoms were detected in any heifer. The carriage of $U$. diversum was detected in 15 animals. The granular vulvovaginitis is commonly associated with $U$. diversum carriage in heifers and cows, but the symptoms of this disease are nonspecific and frequently may be associated with other diseases, for example with bovine rhinotracheitis, that is very widespread in cattle. No association was detected between granular vulvovaginitis symptoms and $U$. diversum carriage in study population.

The effectiveness of fertilization was estimated in all heifers. The average number of inseminations leads to fertilization in heifers without carriage was 1.2 , but in infected heifers it was 1.9, and the difference between two groups was statistically significant $(\mathrm{t}=0.36$; $\mathrm{p}<0.002)$. The fertilization failure was more frequent in heifers with $U$. diversum carriage. Twelve heifers from this group were fertilised at first insemination, two heifers in the same group were fertilized at second insemination and one heifer was inseminated six times before fertilization. Into the group without $U$. diversum carriage all but one of heifers were fertilized in the first insemination an one heifer in second insemination.

The loss in fertilization effectiveness leads to the economic burden in dairy farms due to costs of repeated inseminations and animal management. The appropriate diagnosis of $U$. diversum carriage in heifest may improve dairy farm productivity.

\section{ANTIBIOTIC-RESISTANT KLEBSIELLA PNEUMONIAE} IN THE GUT MICROBIOTA OF HEALTHY INDIVIDUALS

E.V. Voitenkova, L.V. Suzhaeva, A.V. Zabrovskaya, L.A. Kaftyreva

St. Petersburg Pasteur Institute, St. Petersburg, Russia

Klebsiella pneumoniae causes a wide range of infectious diseases including pneumonia, urinary tract infections, bacteremia and liver abscesses. Previously, it was believed that $K$. pneumoniae can cause serious infections primarily in people with decreased immunity, but the recent emergence and spread of hypervirulent strains has increased the number of people susceptible to these infections, including healthy ones. In addition, strains of K. pneumo- niae are becoming more resistant to antibiotics, which creates special difficulties in treatment. Strains of the genus Klebsiella quite often colonize the mucous membrane of the distal gastrointestinal tract of children and adults, being part of the gut microbiota.

The aim of this study was to reveal frequency of occurrence of $K$. pneumoniae in intestinal microbiota of clinically healthy adults and children and to define antimicrobial susceptibility of isolated strains.

The microbiota content of 180 people aged from 1 month to 65 years was studied by quantative bacteriological method according to OST 91500.11.0004-2003 "Protocol of management of patients. Intestinal dysbacteriosis." K. pneumoniae strains were isolated in $25.0 \%$ (95\% CI:19.2-31.8) of samples in quantaties exceeding $10^{5} \mathrm{CFU} / \mathrm{g}$. The susceptibility of these strains to 7 groups of antibiotics (penicillins combined with inhibitors, cephalosporins, quinolones, aminoglycosides, tetracycline, chloramphenicol, nitrofurans) was studied by disc diffusion method. $51.1 \%$ (95\% CI:37.0-65.0) of the isolates were resistant to one or more antimicrobials. Multiple resistance (resistance to 3 or more classes) was found in $11.0 \%(95 \%$ CI:4.8-23.5) isolates. The highest resistance was observed to amoxicilline/clavulanic acid (31.1\% of strains), the lowest - to amikacin (4.4\% of strains). No strains resistant to carbapenems were found. Resistance to other antimicrobials ranged from $8.9 \%$ (chloramphenicol) to $22.2 \%$ (gentamicin).

The study showed that the intestinal microbiota of every fourth clinically healthy person contains strains of $K$. pneumoniae, half of which are resistant to one or more antimicrobials. At the same time, more than $10.0 \%$ of $K$. pneumoniae strains isolated from healthy people have multiple antibiotic resistance. Such strains can serve as a reservoir of determinants of resistance to other enterobacteria, including pathogens of acute intestinal infections.

\subsection{3} doi: $10.15789 / 2220-7619-2018-4-9.33$

\section{ANTIMICROBIAL RESISTANCE MECHANISMS IN BACTERIA STRAINS ISOLATED FROM FARM ANIMALS}

\section{A.V. Zabrovskaya, S.A. Egorova \\ St. Petersburg Pasteur Institute, St. Petersburg, Russia}

The aim of the work was to study the quinolone and $\beta$-lactame resistance mechanisms in Salmonella and opportunistic bacteria strains isolated from farm animals.

We determined the quinolones and $\beta$-lactams susceptibility and resistance mechanisms in 482 Salmonella and 144 strains of opportunistic bacteria (E. coli, Klebsiella spp.).

For 6 Salmonella strains (3S. Enteritidis and 3 $S$. Infantis), resistant to fluoroquinolones the mutations in the QRDR region of gyrA gene were detected by amplification and sequencing of this DNA region (Kosyreva et al., 2012). The extended-spectrum $\beta$-lactamases (molecular classes A and C) were determined by PCR with specific primers (Dallenne et al., 2010). 132 strains of Salmonella were resistant to quinolones $(27.4 \%), 41$ of them $(8.5 \%)$ had high level resistance to ciprofloxacin (MIC 6-32.0 mg/1). Sequencing of the gyrA of some resistant Salmonella isolates have been identified three types of single point mutations. In two $S$. Enteritidis the mutation was noted in 83 position (Serine replacement by Phenylalanine), in one strain - in 87 position (Asparagine replacement by Glycine). Three $S$. Infantis strains had the replacement of Asparagine by Tyrosine in 87 position. 